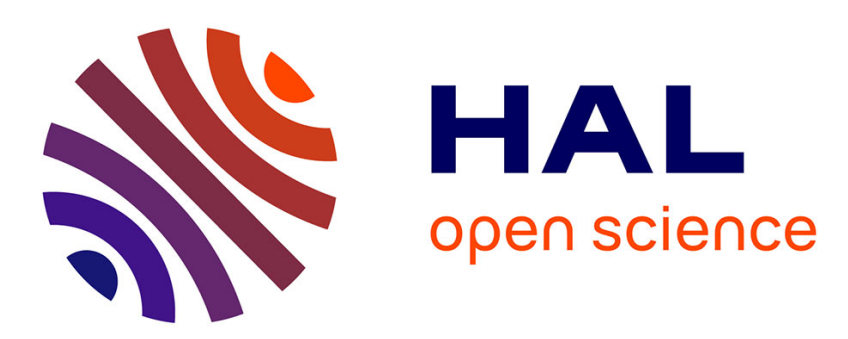

\title{
Towards simulation at picometer-scale resolution: Revisiting inversion domain boundaries in GaN
}

Frédéric Lançon, Luigi Genovese, J. Eymery

\section{To cite this version:}

Frédéric Lançon, Luigi Genovese, J. Eymery. Towards simulation at picometer-scale resolution: Revisiting inversion domain boundaries in GaN. Physical Review B: Condensed Matter and Materials Physics (1998-2015), 2018, 98 (16), pp.165306. 10.1103/PhysRevB.98.165306 . hal-01986656

\section{HAL Id: hal-01986656 https://hal.science/hal-01986656}

Submitted on 14 Mar 2019

HAL is a multi-disciplinary open access archive for the deposit and dissemination of scientific research documents, whether they are published or not. The documents may come from teaching and research institutions in France or abroad, or from public or private research centers.
L'archive ouverte pluridisciplinaire HAL, est destinée au dépôt et à la diffusion de documents scientifiques de niveau recherche, publiés ou non, émanant des établissements d'enseignement et de recherche français ou étrangers, des laboratoires publics ou privés. 


\title{
Towards simulation at picometer-scale resolution: revisiting inversion domain boundaries in $\mathrm{GaN}$
}

\author{
Frédéric Lançon, ${ }^{1, *}$ Luigi Genovese, ${ }^{1}$ and Joël Eymery ${ }^{1}$ \\ ${ }^{1}$ Univ. Grenoble Alpes (UGA), INAC-MEM, F-38000 Grenoble, France \\ CEA, INAC-MEM, L_Sim, F-38054 Grenoble, France
}

(Dated: March 1, 2019)

\begin{abstract}
Motivated by recent high resolution results on the inversion domain boundaries (IDB) in gallium nitride, we refine by $a b$ initio DFT calculations the well established atomic model IDB* derived by Northrup et al. This allows us to recover these experimental results obtained by coherent x-ray diffraction and showing small additional shifts of the polarity domains, in particular $8 \mathrm{pm}$ shift along the hexagonal direction. The influence of boundary conditions and electrostatic fields (IDB-IDB and IDB-surface interactions) on the results and the existence of metastable solutions is carefully discussed to stress the accuracy of the method. These results demonstrate a cross-talk between advanced characterization tools and state-of-the-art $a b$ initio calculations that opens perspectives for the structural analysis of defects in the picometer range.
\end{abstract}

Introduction - Precision in DFT calculations for solid-state physics has been recently studied ${ }^{1}$ and a very good reproducibility of the results has been reported among several current codes. For instance, a reproducibility of $1 \mathrm{pm}$ has been found on the different calculated lattice parameters of silicon. Yet, this precision obtained in the calculation corresponds to an accuracy of $6 \mathrm{pm}$ with respect to the experimental value ${ }^{1}$. We intend here to get a better agreement in the accuracy of geometrical characteristics of gallium nitride, a widely studied compound of technological importance. Challenged by recent experimental results ${ }^{2}$ on very common planar defects in GaN material, we have conducted a detailed analysis of them. Gallium-nitride crystal is a wurtzitelike structure, i.e. a hexagonal lattice with one fourth of the $\mathrm{Ga}-\mathrm{N}$ bonds oriented along the hexagonal-symmetry axis. Inversion domain boundaries (IDB) are interfaces between crystalline polarity domains, which can be noted $-c$ and $+c$. These domains have their hexagonal axis in common, but have opposite orientations for their $\mathrm{Ga}-$ $\mathrm{N}$ bonds parallel to it. By convention, in $+c$ domains these $\mathrm{Ga} \rightarrow \mathrm{N}$ bonds are oriented along [ $\left[\begin{array}{llll}0 & 0 & 0 & 1\end{array}\right]$ of the

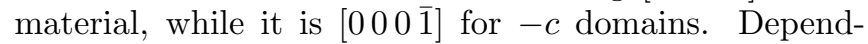
ing on the experimental conditions, a given type of domain is usually preferably produced during the crystal growth. The growing surface perpendicular to $\left[\begin{array}{llll}0 & 0 & 0 & 1\end{array}\right]$ is either Ga terminated for $+c$ domains or $\mathrm{N}$ terminated for $-c$. However, both domain types separated by IDB frequently coexist in thin films ${ }^{3-7}$ and in wires ${ }^{8,9}$. It usually corresponds to detrimental defects in the materials, but periodic alternation of domains is garnering interest in a wide range of devices such as those for non linear frequency conversion ${ }^{10-12}$.

IDB structures are usually studied by electron microscopy ${ }^{3-5,7,9,13}$. These studies confirm the pioneering model for IDB* introduced by Northrup et al ${ }^{14}$, who determined its structure and stability with $a b$ initio DFT calculations. This model gets the lowest energy of possible structures by keeping hetero first-neighbor bonds at the cost of some bond-angle distortions at the boundary.

It provides a characteristic shift of the Ga atomic layers by $c / 8$ along the $\left[\begin{array}{llll}0 & 0 & 0 & 1\end{array}\right]$ direction, which is measured by high resolution transmission electron microscopy ${ }^{4,7}$. Indeed, electron microscopy enhances the contrast of the heavier Ga-atoms with respect to the lighter $\mathrm{N}$-atoms. The IDB have been shown to emit light ${ }^{15,16}$ and the emission properties of the IDB* structure have been calculated, again by DFT calculation ${ }^{17}$, confirming its theoretical basis.

However, experimental advances are now pushing the structure characterizations of boundaries toward the "picoscopic"-scale description of condensed matter ${ }^{2,18}$ and this can challenge the actual IDB* model. In particular, by considering IDB buried in large and long GaN rods obtained by MOVPE (metal-organic vapor phase epitaxy), Labat et al. ${ }^{2}$ found an extra shift of $8 \mathrm{pm} \pm 1 \mathrm{pm}$ to the $c / 8$ shift of the Ga atomic layers crossing the IDB* from the $-c$ domain to the $+c$ one. The authors suggested a segregation of silicon atoms at the interface as a possible explanation of this apparent discrepancy with respect to the usual model of the IDB. Thus it is worth checking in details this model to exclude such possible external explanations of discrepancies. Previous DFT calculation of the GaN IDB* in Ref. 17 gives a hint that this accuracy is achievable, since the deviations of the calculated lattice parameters are there $\Delta a=-2.8 \mathrm{pm}(-0.9 \%)$ and $\Delta c=-4.7 \mathrm{pm}(-0.9 \%)$ compared to the experimental results of Ref. 19. In this paper, we revisit the IDB* model by new ab initio calculations with a larger number of atoms and high energy precision. It will provide a special focus on these new accurate experimental results and allow discussing the intrinsic effect of the IDB with respect to doping distorsions. 
Setup - Taking into account the electronic density in its ground-state, DFT codes are now well established tools to calculate atomic properties for an increasing number of materials ${ }^{1,20}$. We have used BigDFT $\operatorname{code}^{21,22}$, which has the particularity of expressing the electronic wave functions with a basis set of localized wavelet functions. This type of basis enables a strong compression of the data, a systematic control of the precision, and an excellent efficiency for parallel calculations. Besides, the code can take into account a variety of boundary conditions $^{23}$, from isolated molecules to periodic crystals. In particular here, this feature allows us to explore systems with either full periodic boundary conditions or free boundary conditions at the surfaces parallel to the IDB. The first case is usual for such calculations and was indeed the case for the previous calculations with 32 atoms in the supercell ${ }^{14}$ or 48 atoms $^{17}$. However in this case, an even number of IDB has to be introduced in the supercell, usally two IDB and two crystalline grains. In the second case, studies with free surfaces parallel to an interface are usually done with full periodic conditions while inserting a vacuum layer between the surfaces ${ }^{20,24}$. The width of the vacuum must minimize as much as possible the interaction through the empty space of the atoms at the surfaces. Instead here, we have used real free boundary conditions perpendicular to the IDB, while keeping periodic conditions in the two other directions. This feature is possible with the Poisson solver included in BigDFT $\operatorname{code}^{23}$. For both types of calculations, fully periodic or with free boundary conditions, 32 to 256 atoms have been considered. A higher focus has been given to the 80 and 128 atom systems to explore their properties.

Hartwigsen-Goedecker-Hutter pseudopotentials ${ }^{25,26}$ (HGH) are used to simulate the interaction of the nucleus and the core electrons with the valence electrons (respectively, three and five electrons per Ga and $\mathrm{N}$ atom here). Calculations are done with Perdew-BurkeErnzerhof (PBE) exchange correlation functional ${ }^{27}$, which is a standard choice for solid-state studies and in particular is suitable for the GaN compounds ${ }^{28}$. The description of the wave functions, and thus the calculation precision, can be systematically improved by decreasing $h_{\text {grid }}$, the real-space grid spacing between the wavelet-function centers. Reasonable values of $h_{\text {grid }}$ must be lower than twice the length parameters of the HGH pseudopotentials ${ }^{29}$. To go further, we have tested the convergence versus $h_{\text {grid }}$ of the energy difference, $\Delta E_{z b-w}$, between the zinc-blende and the wurtzite gallium-nitride crystals. The calculated limit at low $h_{\text {grid }}$ of this quantity is $\Delta E_{z b-w}=6.73 \mathrm{meV}$ per atom (see figure $\mathrm{S} 2$ in Supporting Information ${ }^{30}$ ). In this work, a value $h_{\text {grid }}=0.02 \mathrm{~nm}$ has been chosen (or lower to be commensurate with the supercell box). It corresponds to a deviation of $0.5 \mathrm{meV} /$ atom on $\Delta E_{z b-w}$.

To study the convergence of the calculations with the $k$-point grid, the energy of the orthogonal cell of the GaN wurtzite crystal has been calculated with an increasing number of $k$-points up to $17 \times 10 \times 10$ (see figure $\mathrm{S} 1$ in
Supporting Information ${ }^{30}$ ). The internal parameter $u$ of the unit cell has been determined for each calculation. Compared to the thinnest $17 \times 10 \times 10$ mesh, a $9 \times 5 \times 5$ Monkhorst Pack mesh gives a discrepancy better than $3 \times 10^{-5} \mathrm{eV} /$ atom. This $k$-point grid is taken as the reference in this article.

The computed cell parameters are:

$a=317.9 \mathrm{pm}, c=518.0 \mathrm{pm}$, and $u=0.377$, i.e. $195.3 \mathrm{pm}$

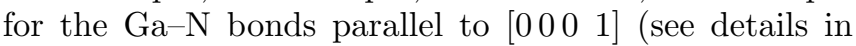
figure S3 in Supporting Information ${ }^{30}$ ). The agreement is excellent with the experimental determination of Ref. 19 with deviations $\Delta a=-0.9 \mathrm{pm}$ and $\Delta c=-0.6 \mathrm{pm}$. This confirms the validity of the approach to get IDB geometry at a picometer resolution.

The IDB* are perpendicular to the $b$ axis. In the calculations a number of unit cells are packed along $b$ on each side of the interface, and a $9 \times 1 \times 5$ mesh of $k$-points is then sufficient. The crystalline grains are thus spanning the $b$-direction, which is taken as our $x$-axis and is equal

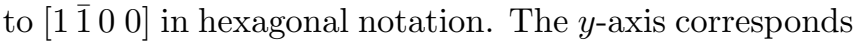
to $\left[\begin{array}{llll}1 & 1 & \overline{2} & 0\end{array}\right]$ ( $a$-axis) and the $z$-axis to [ [ $\left.\begin{array}{llll}0 & 0 & 0 & 1\end{array}\right]$ (c-axis).

Results - Two atomic configurations have been considered as starting structures for minimizing the system energy. First, the structure as described by Northrup et $a l^{14}$ where the Ga layers perpendicular to the $\left[\begin{array}{llll}0 & 0 & 0 & 1\end{array}\right]$ direction in one grain are aligned with nitrogen layers in the second grain. Second, a similar configuration except that an extra shift $\delta z=8 \mathrm{pm}$ is added between the $+c$ and $-c$ domains. This particular value of $\delta z$ corresponds to the experimental results of Ref. 2. These setups have been used for systems with increasing grain sizes along $x$ up to a total of 256 atoms, i.e. 64 (1 100$)$-layers in each grain. For all grain sizes and after energy minimization, the lowest energy has always been obtained when the initial shift is $8 \mathrm{pm}$. The final optimum shift is $\delta z=8.2 \pm 0.1 \mathrm{pm}$ in agreement with the Bragg imaging results ${ }^{2}$ obtained by coherent X-ray diffraction (see figures 4 and 5 in the appendix). A metastable state is reached by the configurations without initial shift. Its final grain shift corresponds actually to a negative shift $\delta z=-1.0 \pm 0.1 \mathrm{pm}$. Other configurations with intermediate initial grain shifts have also been tested and all of them have fallen in one or the other of these two minima. Figure 1 shows the structure and electron density of the IDB for both states in an overlay mode to appreciate the very small change between them (see also figure S6 to $\mathrm{S} 8$ in Supporting Information ${ }^{30}$ ). It evidences how big the challenge is to measure it experimentally. Interferometric approaches, such as coherent x-ray diffraction and electron holography, seem indeed well suited to get these small variations in atomic structures.

Each calculation has been performed at constant supercell sizes and, in particular along $y$ and $z$, the $a$ and $c$ lattice parameters have been set to the perfect crystal parameters to mimic infinitely-separated interfaces. However, fixed size along $x$ forbids free grain shifts perpendicular to the interface. Therefore, the grain separation at the interface may not be the optimum distance. 

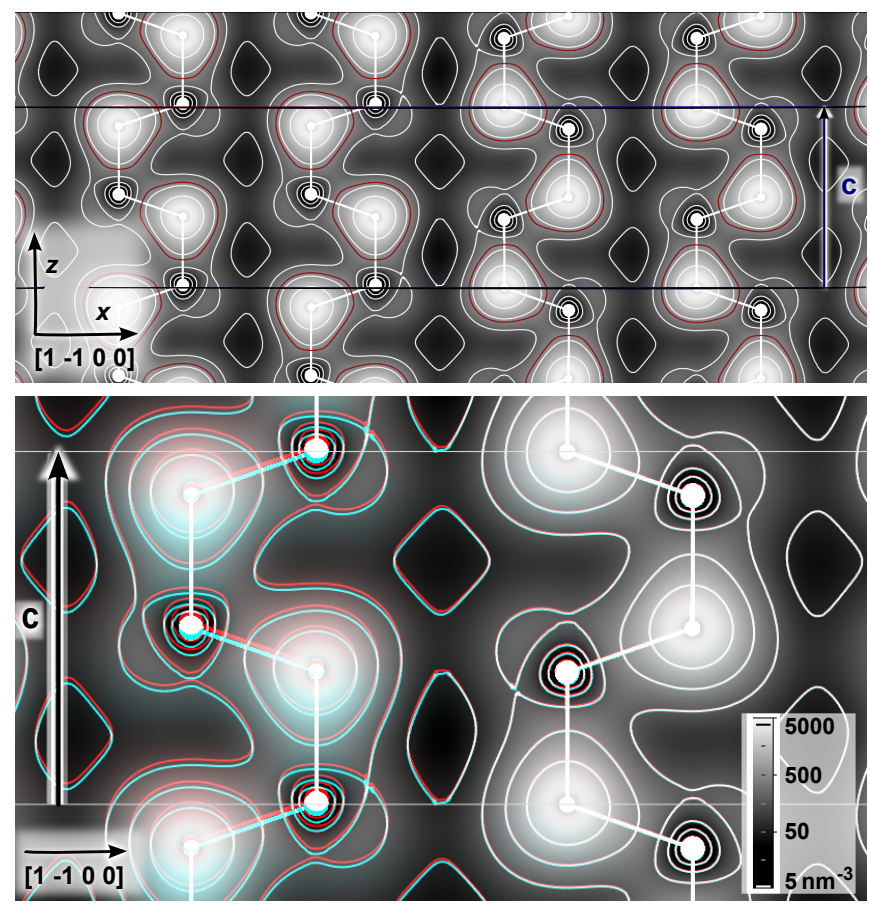

FIG. 1. Density plot of the valence-electron density at the inversion domain boundary between $+c$ and $-c$ domains (respectively at left and right). In Supporting Information ${ }^{30}$, figure S7 shows the plane location of the density cross-section (available at [URL will be inserted by publisher]). White and black represent respectively the highest and the lowest electron density. Valence electrons are mostly located around nitrogen atoms. Top: Density of the ground state. Low and high density regions are separated by the red curves that are the isovalue lines corresponding to the average density $N_{\mathrm{e}} / V=353 \mathrm{~nm}^{-3}$, where $N_{\mathrm{e}}$ is the total number of valence electrons in the supercell of volume $V$. Bottom: The stable and the metastable structures are both shown, respectively as red and cyan images added together. Where densities are similar, added red and cyan give white, gray or black. The difference of density distributions and the shift of the $+c$ domains are therefore highlighted by the red color coming from the stable configuration and the cyan color coming from the metastable one. For the overlaying, the two $-c$ domains coincide far from the interface, giving them a common origin. The lattice parameter $\mathbf{c}$ is indicated and its modulus equal to $518 \mathrm{pm}$ gives the scale.

Thus different configurations have been constructed with different shifts $\delta L$ by adding or removing space between the domains ( $\delta L=0$ corresponding to the direct inversion of half of the bulk structure when creating the IDB). These configurations have been relaxed with the above protocol. Figure 2 shows the interface energy $E_{\mathrm{i}}$ versus $\delta L$. For both stable and metastable states, and for all grain sizes studied, we found that the optimal values are $\delta L<2 \mathrm{pm}$. However, because of long range interactions between the two interfaces of the periodic configurations, the bulk part of the crystals may be strained. At the energy minimum, a careful analysis of this strain gives a value of $\varepsilon_{x x}=+4 \times 10^{-4}$ for the 80 atom configuration

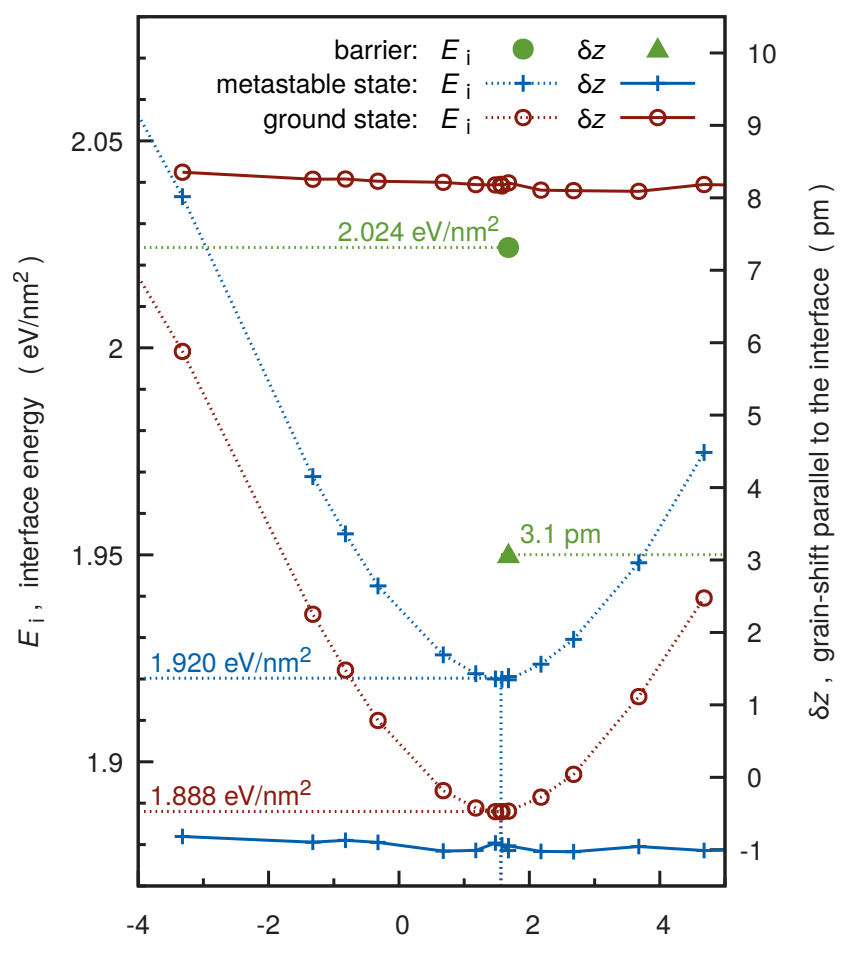

$\delta L$, space added perpendicularly to the interface ( $\mathrm{pm}$ )

FIG. 2. Interface energy $E_{\mathrm{i}}$ (dashed lines and left axis) and domain shift $\delta z$ along [ $\left[\begin{array}{llll}0 & 0 & 0 & 1\end{array}\right]$ (solid lines and right axis) versus spacing $\delta L$ perpendicular to the interfaces. $\delta L$ is the initial deviation at the interface of the interplanar distance $d_{1 \overline{1} 0} 0$. Periodic boundary conditions have been used and data correspond to a 80 atom configuration. In such a periodic system with 2 IDB, the interface energy $E_{\mathrm{i}}$ is, per surface unit, half the difference between the total potential energy of the configuration and the energy of a crystalline configuration with the same number of atoms. Domain shifts $\delta z=z_{\mathrm{Ga}}-z_{\mathrm{N}}$ are calculated as coordinate differences between central $\mathrm{Ga}$ atoms of the $+c$ domain and central nitrogen atoms of the facing layer in the $-c$ domain (see figure 4 in the appendix). Filled circle and triangle correspond respectively to $E_{\mathrm{i}}$ and $\Delta z$ for the barrier between the two minima.

whose distance between interfaces is $2.75 \mathrm{~nm}$. Thus the IDB-IDB interaction is repulsive and its effect on the lattice deformation is small for this size. Nevertheless, for the grain shift perpendicular to the IDB, the uncertainty is mainly due to this strain effect. In the value of $\delta L$, the share of the elastic expansion $\varepsilon_{x x}$ on one hand, and that of the optimal shift $\delta x$ at the IDB on the other hand, are discussed below in the analysis of the boundary-condition effects.

The formation energy $E_{i}$ of the most stable IDB structure is $1.89 \mathrm{eV} / \mathrm{nm}^{2}$, while for the metastable state it is $1.92 \mathrm{eV} / \mathrm{nm}^{2}$. Considering the differences in the DFT methods, this last value is close to the $E_{i}$ value $2.0 \mathrm{eV} / \mathrm{nm}^{2}$ in Ref. 17, where the metastable state has presumably been obtained since no initial shift $\delta z$ was considered. Even if the energy difference per $\mathrm{nm}^{2}$ is small 
between the metastable state and the ground state, it has to be integrated on the total area of the interface to appreciate the respective stability.

Equally important is the energy barrier between the two states and, more precisely, the saddle point of the surface energy in configuration space. First, eight intermediate configurations have been constructed by linear interpolation between the two minima and thus corresponding to intermediate grain shifts $\delta z=n$ pm of the two crystalline grains. Second, DIIS calculations (direct inversion of iterative subspace ${ }^{31}$ ) have been performed with BigDFT code for a geometry optimization ${ }^{32}$ of each of these initial configurations. Here, DIIS calculations correspond to structures relaxations toward close stationary points of the energy surface, usually minima but also saddle points. Indeed, all configurations have converged toward one or the other previous minima, except for the initial $\delta z=3 \mathrm{pm}$, which has led to a saddle point at $0.14 \mathrm{eV} / \mathrm{nm}^{2}$ above the lowest minimum and a final shift $\delta z=3.1 \pm 0.1 \mathrm{pm}$. See in Figure S14 (Supporting Information $^{30}$ ) the atomic configuration and its unstable direction along which the energy second derivative is negative. The energy and the final shift $\delta z$ of the saddlepoint configuration are also plotted in Fig. 2 .

LDA exchange-correlation functional - We test here the strength of our results with respect to the choice of the exchange-correlation functional, which is the key approximation in any DFT calculations. Like PBE, the local density approximation (LDA) is also a standard choice for compound calculations. It usually results in an underestimation of the experimental lattice parameters by a few percent. The lattice parameters calculated with LDA are also lower than those obtained with PBE, and somehow LDA and PBE give the extreme values of the DFT results ${ }^{28}$. For this reason, we have also tested the characteristics of the IDB, when calculated with the LDA functional.

Except for the functional change, the GaN wurtzite lattice parameters have been determined with the same calculation parameters as above and we find: $a_{\mathrm{LDA}}=306.4 \mathrm{pm}, c_{\mathrm{LDA}}=499.8 \mathrm{pm}$, and $u_{\mathrm{LDA}}=0.377$ (see also table S1 in Supporting Information ${ }^{30}$ ). Parameters $a_{\mathrm{LDA}}$ and $c_{\mathrm{LDA}}$ are indeed a few percent lower than both the PBE and the experimental results.

Starting now with the lattice structure corresponding to LDA and repeating the above protocol to build and relax the configurations containing the IDB defects, we obtain again two minima and one barrier. The shifts $\delta z_{\mathrm{LDA}}$ equal $8.2 \pm 0.1 \mathrm{pm},-0.8 \pm 0.1 \mathrm{pm}$, and $3.0 \pm 0.1 \mathrm{pm}$, respectively for the ground state, the metastable state and the barrier (compare figure 4 and figure S9 in Supporting Information $^{30}$ ). Similarly to the PBE result, we found an energy barrier above the ground state of $0.14 \mathrm{eV} / \mathrm{nm}^{2}$. However we found higher defect energies at the minima than for PBE: $3.38 \mathrm{eV} / \mathrm{nm}^{2}$ and $3.43 \mathrm{eV} / \mathrm{nm}^{2}$.

The fact that two different and well established exchange-correlation functionals both lead to double minima for the IDB, and especially a ground state with

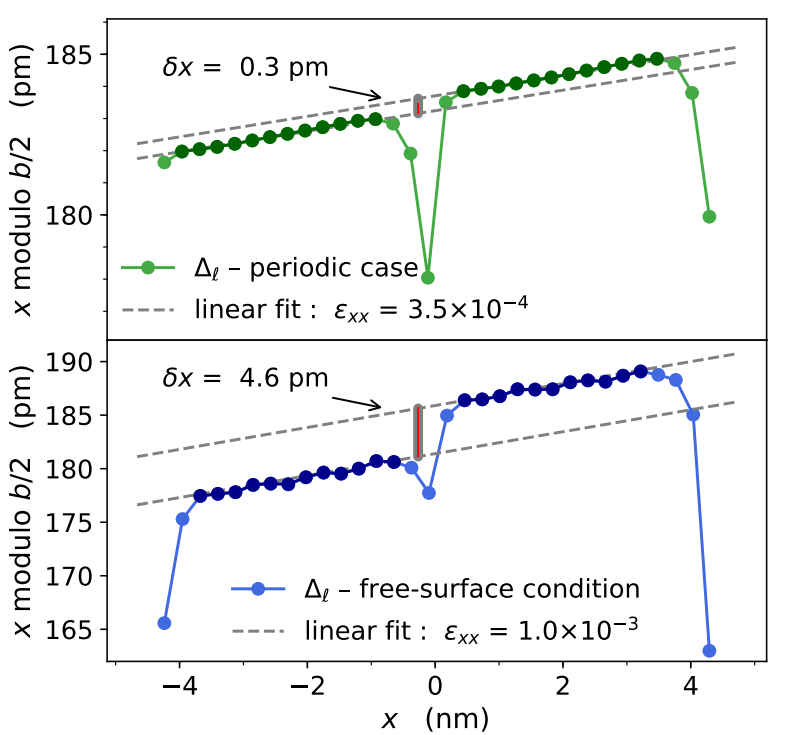

FIG. 3. Atomic shift $\Delta_{\ell}$ associated with the interplane distance $b / 2$ in the direction $x$, i.e. [ $\left[\begin{array}{lll}1 & \overline{1} & 0\end{array}\right]$. The atomic configurations have 128 atoms. Top: two IDB with periodic boundary conditions. Bottom: one IDB and two free surfaces. The plots are centered on a IDB and the vertical origins are arbitrary. The grain shift $\delta x$ and the strain $\varepsilon_{x x}$ are extracted from the linear interpolations through the data of each domain (darker disks). One Ga $x y$-layer is used in the calculation. The deviating values of $\Delta_{\ell}$ in the middle and at the extremities correspond to the atomic relaxations at the IDB and at the free surfaces.

a shift $\delta z$ in agreement with the experiment, reinforces our conclusions.

Boundary conditions - Electrostatic effects - To assess the role of the boundary conditions on our results, calculations with free boundary conditions have also been carried out with the PBE functional. Instead of the two interacting IDB - due to the artificial periodicity perpendicular to the interfaces - there are now possible interactions between one IDB and the free surfaces parallel to it (configurations shown in figures S12 and S13 in Supporting Information ${ }^{30}$ ). The initial surfaces has been obtained by truncating the lattice perpendicularly

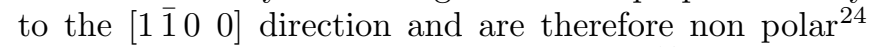
(see figure S10 in Supporting Information ${ }^{30}$ ). No external atoms have been added at these $(1 \overline{1} 00)$-surfaces to passivate them, in order to get the clean-surface effects. All the atoms have been allowed to relax. The calculated relaxations of these bare surfaces are in agreement with those of previous studies ${ }^{20,33-36}$, with a contraction and a rotation of the Ga-N bonds at the surface, the Ga atoms being displaced inward (see also table S2 and figure S11 in Supporting Information ${ }^{30}$ ). For all configurations studied from 32 atoms up to 128 atoms, the IDB has two stable configurations. The domains are shifted 
by $\delta z=8 \mathrm{pm}$ in those with the lowest energy and by $-1 \mathrm{pm}$ in the metastable ones. These results are in agreement with those obtained above with periodic boundary conditions, and thus with the experimental results.

Because of the IDB interactions and the resulting strain, we have seen above that the ideal domain separation at the IDB, or equivalently the shift $\delta x$ to the crystalline interplanar distance $d_{1 \overline{1} 00}$, is more difficult to precisely determine ${ }^{37}$ than the shift $\delta z$. Moreover, with free surfaces parallel to the IDB, we don't have the computer box periodicity to fix the total length of the configuration along $x$, and we can't plot energy curves like in Fig. 2. Thus, to carefully measure $\delta x$, we introduce a phase method where the atomic positions are compared to a reference crystal. This method is analog to the calculation of the hull function describing atomic displacements when two incommensurate structures are interacting ${ }^{18}$. It is also similar to the geometrical phase analysis $^{38-40}$, used in high resolution electron microscopy to measure displacement and strain fields in materials. In this last method however, inputs are images and the variation from a lattice reference is processed through Fourier space. When data are atomic coordinates given by computations, an atomic phase can be simply calculated for each atom $\ell$ as $\Phi_{\ell}=2 \pi\left(\mathbf{r}_{\ell} \cdot \mathbf{q} \bmod 1\right)$, where $\mathbf{q}$ is a chosen reciprocal vector of the reference lattice and $\mathbf{r}_{\ell}$ is the position of atom $\ell$. Here, to get the atomic displacements in length unit along direction $\mathbf{q}$, we plot $\Delta_{\ell}=\mathbf{u}_{\ell}$ $\bmod (1 /\|\mathbf{q}\|)$ versus $\mathbf{u}_{\ell}=\mathbf{r}_{\ell} \cdot \mathbf{q} /\|\mathbf{q}\|$. Note that $1 /\|\mathbf{q}\|$ is the interplanar distance corresponding to $\mathbf{q}$. Thus, the atomic phases and the atomic shifts associated with $\mathbf{q}$ are related by $\Phi_{\ell}=\Delta_{\ell} 2 \pi /\|\mathbf{q}\|$.

In our particular case, the value $\delta x$ as well as the elastic strain in the crystal grains can be extracted from the plot associated with $\|\mathbf{q}\|=2 / b$ in the $x$-direction. This is shown in figure 3 for the periodic system and for the system with free-surfaces. In both cases the crystalline grains are in tension. However, the strain $\varepsilon_{x x}$ is 3 times larger in the free-surface case, and thus the mixed interactions between surfaces and IDB are larger than the interactions between the IDB themselves. We have not enough results to deduce the variations of $\varepsilon_{x x}$ versus distances, but we have indeed checked the logical result that it decreases with the system sizes. With surfaces, we found a larger grain shift $\delta x$ than with periodic conditions (see also figure S5 in Supporting Information ${ }^{30}$ ). Taking into account that these systems are under a larger stress, the periodic boundary conditions give a more reliable result. We can conclude that the domain shift $\delta x$ is close to zero. This is indeed in agreement with the experimental results of Ref. 2 where it is concluded from the value of their error bar that $\delta x<4 \mathrm{pm}$. Note however that the value $\delta x$ obtained with free surfaces is due to electrostatic interactions between the IDB and the free surfaces and thus, could be present in thin nanowires where IDB can be close to the surface ${ }^{8,9}$.

Conclusion - Several experimental techniques are under development to reach the picometer resolution and to study extended defects, especially in semiconductors where they play an important role on the physical properties of electronic and optoelectronic devices. These techniques are mostly based on the interference of coherent $\mathrm{x}$-ray ${ }^{2,41}$ and electron diffraction ${ }^{42}$ beams, or on the scanning of transmitted electron beam by correcting both spherical aberrations and distortions ${ }^{7,43}$. As in the calculation, they require a portion of good crystal to provide a reference in order to estimate the lattice displacements induced by the presence of extended defects.

In parallel to these experimental works, ab initio calculations should now attempt to reach a similar goal to enable cross-talks between experiment and theory. Along this line, we have revisited here the theoretical basis of IDB* $^{*}$, focusing on the detail of its structure, and this resolution can indeed be reached in gallium nitride. To achieve this accuracy, which is validated by experimental results, we have taken advantage of the high precision achieved by the DFT code $^{22}$ and by a favorable behavior of simulations for the GaN compound. The high precision of the calculations comes from different characteristics of the program, in particular the type of wavelet basis and their implementation ${ }^{21}$. The mark of these ingredients is the regularity of the energy and phase curves (Fig 2 and 3 ) with sub-picometer input variations (see also figure S5 in Supporting Information ${ }^{30}$ ).

For the well-established IDB* structure, we found that the GaN inversion domains are subjected to a positive or a negative extra lattice shift parallel to the hexagonal axis leading to two states, respectively $\mathrm{IDB}^{+}$and $\mathrm{IDB}^{-}$. The positive shift $\delta z$ leads to the ground state and its value of $8 \mathrm{pm}$ is in agreement with the reported experimental results ${ }^{2}$. We have carefully checked the effects of the boundary conditions. Like the size of the configurations, they do not change the ground state status of the two minima, neither their respective values of $\delta z$. Note that in simulations with Tersoff-Brenner potentials for GaN, i.e. without electronic density taken into account, only one minimum had been found ${ }^{2}$. GaN wurtzite is a strongly polarized compound and we could expect a similar behavior for IDB in wurtzite $\mathrm{ZnO}$, which has very similar physical properties.

While our various calculations of $\delta z$ converge, the domain shift $\delta x$, perpendicular to the interface, is more dependent on the boundary conditions. The interactions between surface and IDB are strong for the configuration sizes studied here, and periodic conditions are recommended, as usual, for this determination. Nevertheless, for thin wires grown by molecular beam epitaxy where IDB can be located very close to the surface, our results with free-surface conditions can be pertinent and predict larger values of $\delta x$ than in the bulk. Determined with periodic conditions, the shift $\delta x$ for the bulk is lower than half a picometer. Experimentally, this value is difficult to measure too, but the interval given ${ }^{2}$ of $\delta x<4 \mathrm{pm}$ is in agreement with our result.

Supplemental Material -

File IDB-supplement-2018.pdf ${ }^{30}$ is available with the 
following information:

(1) $k$-point grid and $h$-grid parameter convergences; (2) lattice parameter determinations; (3) grain-shift $\delta x$ convergence; (4) plots of the atomic configuration and the electron density of $\mathrm{IDB}^{+} ;(5)$ grain-shift $\delta z$ with LDA; (6) surface relaxation; (7) collection of ball-and-stick models; (8) Hessian matrix calculation; (9) list and description of the atomic-configuration files that are also available as Supplemental Material.

\section{Appendix: Grain shift $\delta z$}

The grain shift $\delta z$ is the main quantity of the system that indicates a discrepancy between the original IDB* model $^{14}$ and our calculation, as well as with the experimental results ${ }^{2}$. It is therefore crucial to calculate it carefully. This could be done by using the phase method introduced above. Indeed, in the same way as the shift $\delta x$ has been calculated, we could calculate $\delta z$ using the $c$ lattice parameter instead of the $b$ one, i.e. with $\Delta_{\ell}=z_{\ell}$ $\bmod c / 2$. However in an atomic row perpendicular to the IDB, the coordinate $z$ varies by a much lower quantity than $c / 2$. Thus plotting $z_{\ell}$ versus $x$ is enough to determine $\delta z$.

Figure 4 shows the atomic coordinates $z_{\ell}$, as well as the global shifts $\delta z$ found for three states: the ground-state $\mathrm{IDB}^{+}$, the metastable state $\mathrm{IDB}^{-}$, and the saddle-point state separating $\mathrm{IDB}^{+}$and $\mathrm{IDB}^{-}$. This figure and figure 5 show the precision achieved by the calculations and the consistency of the results with the system size. More generally, figures 3 and 4 show the atomic relaxations at the interface respectively along $x$ and $z$. No relaxation occurs along $y$.

\section{ACKNOWLEDGMENTS}

The authors thank Damien Caliste for his open software $V_{-} \mathrm{Sim}$, which has allowed us to visualize the atomic configurations and the electronic densities. They also thank Thierry Deutsch for useful discussions and suggestions.

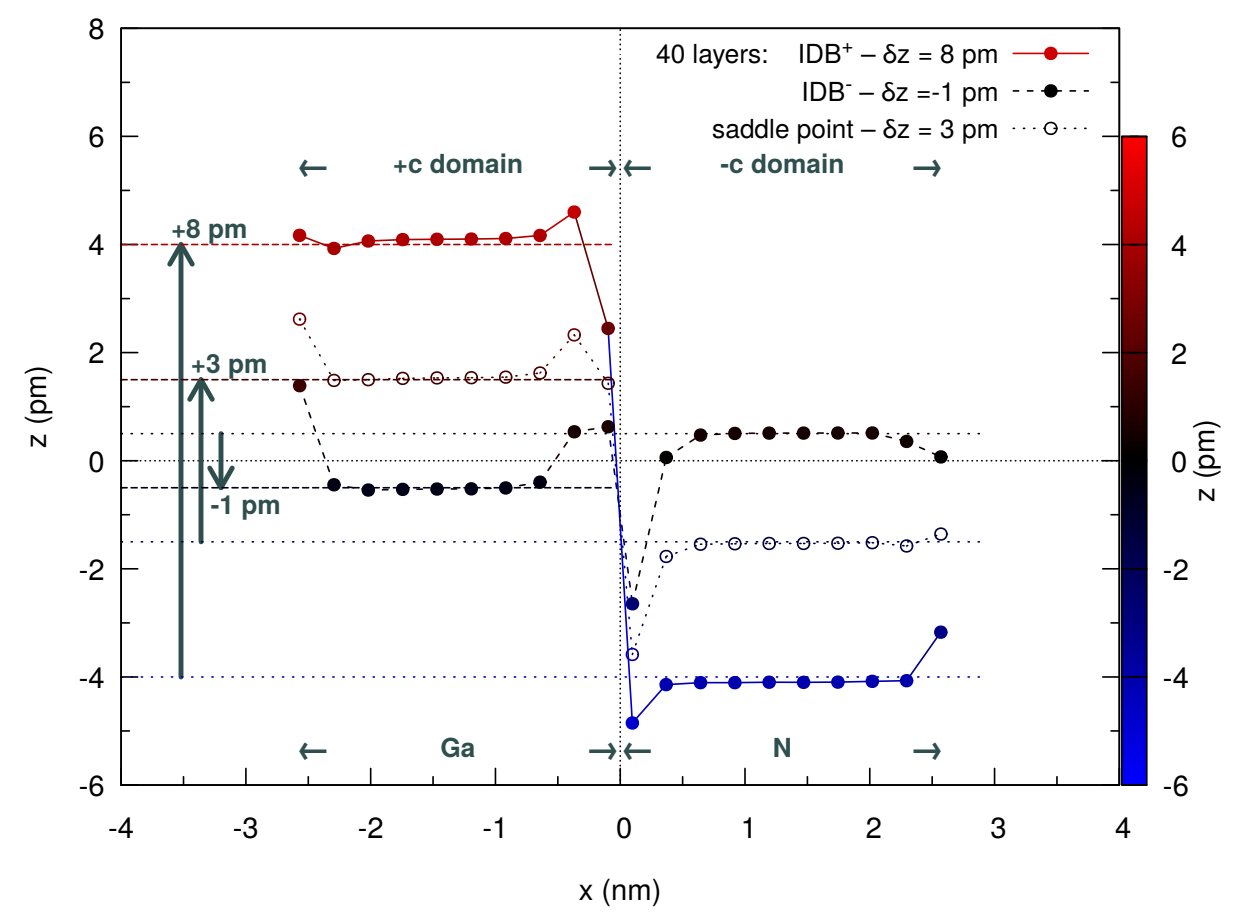

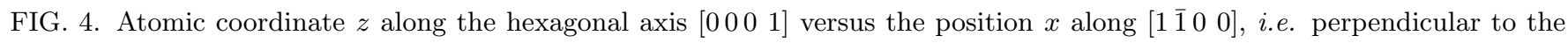
interface. One atomic layer crossing orthogonally the interface is considered for this graph. It consists of Ga atoms on one side ( $+\mathrm{c}$ domain) and $\mathrm{N}$ atoms on the other side (-c domain). The results are for three 80 -atom configurations (i.e. with 40 layers parallel to the IDB): the ground state IDB $^{+}$, the metastable state IDB $^{-}$, and the saddle-point state. They correspond respectively to domain shifts $\delta z$ equal to $+8.2 \mathrm{pm},-1.0 \mathrm{pm}$, and $+3.1 \mathrm{pm}$. 


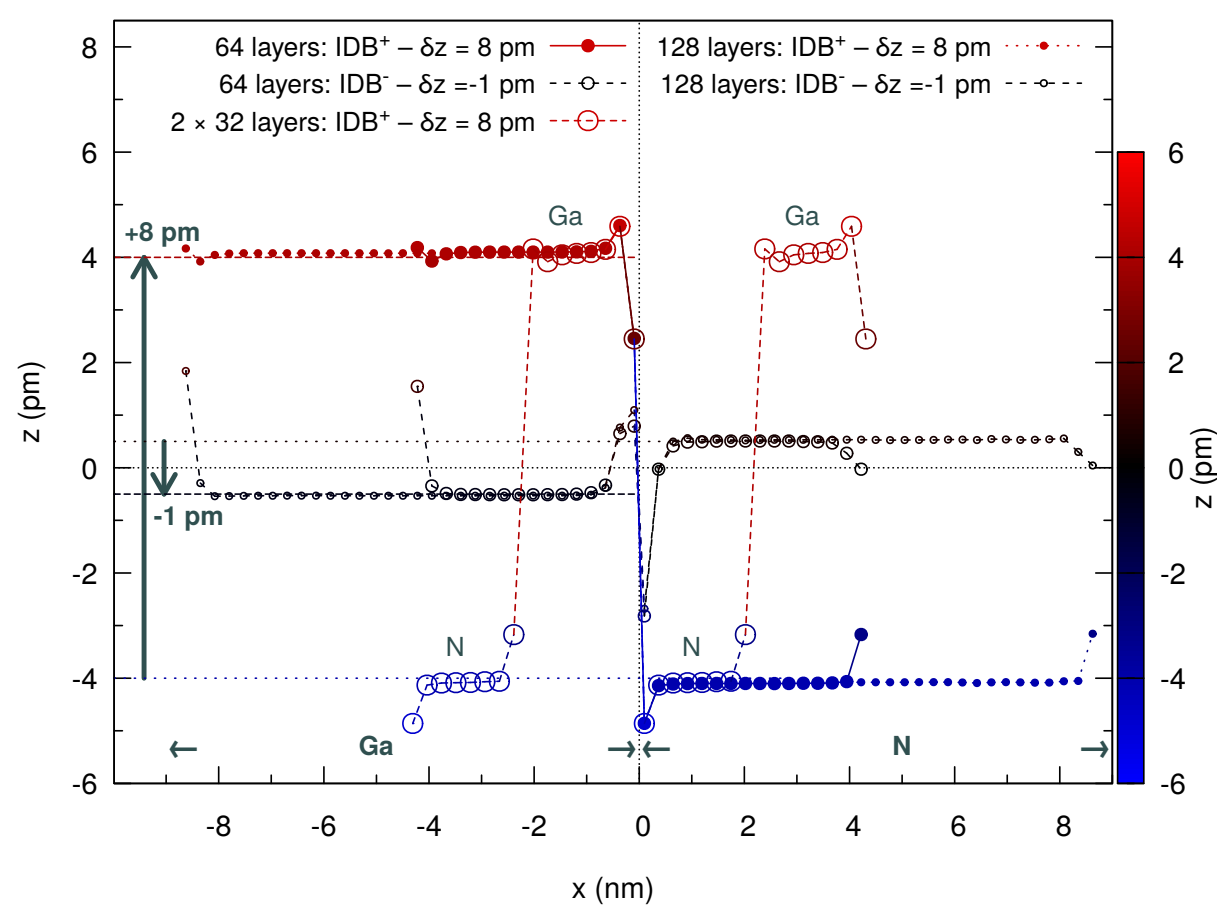

FIG. 5. Summary of the results for 128-atom and 256-atom configurations (64 and 128 layers respectively) showing the atomic coordinate $z$ along the hexagonal axis versus the position $x$ perpendicular to the interface. One special configuration contains four instead of two IDB $(2 \times 32$ layers $)$, and thus four domains. The coherence of the results among different system sizes is thus validated.

* Frederic.Lancon@cea.fr

1 Kurt Lejaeghere, Gustav Bihlmayer, Torbjoern Bjoerkman, Peter Blaha, Stefan Bluegel, Volker Blum, Damien Caliste, Ivano E. Castelli, Stewart J. Clark, Andrea Dal Corso, Stefano de Gironcoli, Thierry Deutsch, John Kay Dewhurst, Igor Di Marco, Claudia Draxl, Marcin Dulak, Olle Eriksson, Jose A. Flores-Livas, Kevin F. Garrity, Luigi Genovese, Paolo Giannozzi, Matteo Giantomassi, Stefan Goedecker, Xavier Gonze, Oscar Granaes, E. K. U. Gross, Andris Gulans, François Gygi, D. R. Hamann, Phil J. Hasnip, N. A. W. Holzwarth, Diana Iusan, Dominik B. Jochym, François Jollet, Daniel Jones, Georg Kresse, Klaus Koepernik, Emine Kuecuekbenli, Yaroslav O. Kvashnin, Inka L. M. Locht, Sven Lubeck, Martijn Marsman, Nicola Marzari, Ulrike Nitzsche, Lars Nordstrom, Taisuke Ozaki, Lorenzo Paulatto, Chris J. Pickard, Ward Poelmans, Matt I. J. Probert, Keith Refson, Manuel Richter, Gian-Marco Rignanese, Santanu Saha, Matthias Scheffler, Martin Schlipf, Karlheinz Schwarz, Sangeeta Sharma, Francesca Tavazza, Patrik Thunstroem, Alexandre Tkatchenko, Marc Torrent, David Vanderbilt, Michiel J. van Setten, Veronique Van Speybroeck, John M. Wills, Jonathan R. Yates, Guo-Xu Zhang, and Stefaan Cottenier, "Reproducibility in density functional theory calculations of solids," Science 351, 1415-U81 (2016).

2 Stéphane Labat, Marie-Ingrid Richard, Maxime Dupraz,
Marc Gailhanou, Guillaume Beutier, Marc Verdier, Francesca Mastropietro, Thomas W. Cornelius, Tobias U. Schülli, Joël Eymery, and Olivier Thomas, "Inversion domain boundaries in GaN wires revealed by coherent bragg imaging," ACS Nano 9, 9210-9216 (2015), http://dx.doi.org/10.1021/acsnano.5b03857.

3 L. T. Romano, J. E. Northrup, and M. A. O'Keefe, "Inversion domains in GaN grown on sapphire," Applied Physics Letters 69, 2394-2396 (1996).

4 J.L. Rouvière, M. Arlery, B. Daudin, G. Feuillet, and O. Briot, "Transmission electron microscopy structural characterisation of GaN layers grown on (0001) sapphire," Materials Science and Engineering: B 50, 61 - 71 (1997).

5 Jean-Luc Rouvière, M. Arlery, and Alain Bourret, "Structural characterisation of GaN layers : influence of polarity and strain release," Institute of Physics Conference Series 157, 173-182 (1997), presented at the Royal Microscopical Society Conference on Microscopy of Semiconducting Materials, 7-10 April 1997. Article reprint from Jean-Luc.

6 A. M. Sanchez, G. Nouet, P. Ruterana, F. J. Pacheco, S. I. Molina, and R. Garcia, "A mechanism for the multiple atomic configurations of inversion domain boundaries in GaN layers grown on Si(111)," Applied Physics Letters 79, 3588-3590 (2001).

7 Fude Liu, Ramon Collazo, Seiji Mita, Zlatko Sitar, Stephen J. Pennycook, and Gerd Duscher, "Direct ob- 
servation of inversion domain boundaries of GaN on csapphire at sub-Ångstrom resolution," Advanced Materials 20, 2162-2165 (2008).

8 X. J. Chen, G. Perillat-Merceroz, D. Sam-Giao, C. Durand, and J. Eymery, "Homoepitaxial growth of catalyst-free GaN wires on N-polar substrates," Applied Physics Letters 97, 151909 (2010), https://doi.org/10.1063/1.3497078.

9 Thomas Auzelle, Benedikt Haas, Martien Den Hertog, Jean-Luc Rouvière, Bruno Daudin, and Bruno Gayral, "Attribution of the $3.45 \mathrm{eV}$ GaN nanowires luminescence to inversion domain boundaries," Applied Physics Letters 107, 051904 (2015).

10 Aref Chowdhury, Hock M. Ng, Manish Bhardwaj, and Nils G. Weimann, "Second-harmonic generation in periodically poled GaN," Applied Physics Letters 83, 1077-1079 (2003), http://dx.doi.org/10.1063/1.1599044.

11 Jennifer Hite, Mark Twigg, Michael Mastro, Jaime Freitas, Jerry Meyer, Igor Vurgaftman, Shawn O'Connor, Nicholas Condon, Fritz Kub, Steven Bowman, and Charles Eddy, "Development of periodically oriented gallium nitride for non-linear optics," Opt. Mater. Express 2, 12031208 (2012).

12 Jennifer K. Hite, Nelson Y. Garces, Ramasis Goswami, Michael A. Mastro, Fritz J. Kub, and Charles R. Eddy Jr., "Selective switching of GaN polarity on Ga-polar GaN using atomic layer deposited $\mathrm{Al}_{2} \mathrm{O}_{3}$," Applied Physics Express 7, 025502 (2014).

13 J.L. Rouvière, M. Arlery, R. Niebuhr, K.H. Bachem, and O. Briot, "Transmission electron microscopy characterization of GaN layers grown by MOCVD on sapphire," Materials Science and Engineering: B 43, 161 - 166 (1997), e-MRS 1996 Spring Meeting, Symposium C: UV, Blue and Green Light Emission from Semi-conductor Materials.

14 John E. Northrup, Jörg Neugebauer, and L. T. Romano, "Inversion domain and stacking mismatch boundaries in GaN," Phys. Rev. Lett. 77, 103-106 (1996).

15 P. J. Schuck, M. D. Mason, R. D. Grober, O. Ambacher, A. P. Lima, C. Miskys, R. Dimitrov, and M. Stutzmann, "Spatially resolved photoluminescence of inversion domain boundaries in GaN-based lateral polarity heterostructures," Applied Physics Letters 79, 952-954 (2001).

16 Ronny Kirste, Ramón Collazo, Gordon Callsen, Markus R. Wagner, Thomas Kure, Juan Sebastian Reparaz, Seji Mita, Jinqiao Xie, Anthony Rice, James Tweedie, Zlatko Sitar, and Axel Hoffmann, "Temperature dependent photoluminescence of lateral polarity junctions of metal organic chemical vapor deposition grown GaN," Journal of Applied Physics 110, 093503 (2011).

17 Vincenzo Fiorentini, "Origin of the efficient light emission from inversion domain boundaries in GaN," Applied Physics Letters 82, 1182-1184 (2003).

18 A. Gautam, C. Ophus, F. Lançon, V. Radmilovic, and U. Dahmen, "Atomic structure characterization of an incommensurate grain boundary," Acta Materialia 61, 5078 - 5086 (2013).

19 M. Leszczynski, H. Teisseyre, T. Suski, I. Grzegory, M. Bockowski, J. Jun, S. Porowski, K. Pakula, J. M. Baranowski, C. T. Foxon, and T. S. Cheng, "Lattice parameters of gallium nitride," Applied Physics Letters 69, 73-75 (1996).

20 Jesús Zúñiga Pérez, Vincent Consonni, Liverios Lymperakis, Xiang Kong, Achim Trampert, Sergio FernándezGarrido, Oliver Brandt, Hubert Renevier, Stacia Keller,
Karine Hestroffer, Markus R. Wagner, Juan Sebastián Reparaz, Fatih Akyol, Siddharth Rajan, Stéphanie Rennesson, Tomás Palacios, and Guy Feuillet, "Polarity in gan and zno: Theory, measurement, growth, and devices," Applied Physics Reviews 3, 041303 (2016), https://doi.org/10.1063/1.4963919.

21 Luigi Genovese, Alexey Neelov, Stefan Goedecker, Thierry Deutsch, Seyed Alireza Ghasemi, Alexander Willand, Damien Caliste, Oded Zilberberg, Mark Rayson, Anders Bergman, and Reinhold Schneider, "Daubechies wavelets as a basis set for density functional pseudopotential calculations," The Journal of Chemical Physics 129, 014109 (2008).

22 BigDFT, "version 1.8," (2016).

23 Alessandro Cerioni, Luigi Genovese, Alessandro Mirone, and Vicente Armando Sole, "Efficient and accurate solver of the three-dimensional screened and unscreened poisson's equation with generic boundary conditions," The Journal of Chemical Physics 137, 134108 (2012).

24 V.M. Bermudez, "The fundamental surface science of wurtzite gallium nitride," Surface Science Reports 72, 147 -315 (2017).

25 S. Goedecker, M. Teter, and J. Hutter, "Separable dualspace gaussian pseudopotentials," Phys. Rev. B 54, 17031710 (1996).

26 C. Hartwigsen, S. Goedecker, and J. Hutter, "Relativistic separable dual-space Gaussian pseudopotentials from H to Rn," Phys. Rev. B 58, 3641-3662 (1998).

27 John P. Perdew, Kieron Burke, and Matthias Ernzerhof, "Generalized gradient approximation made simple," Phys. Rev. Lett. 77, 3865-3868 (1996).

28 Philipp Haas, Fabien Tran, and Peter Blaha, "Calculation of the lattice constant of solids with semilocal functionals," Phys. Rev. B 79, 085104 (2009).

29 Luigi Genovese and Thierry Deutsch, "Multipolepreserving quadratures for the discretization of functions in real-space electronic structure calculations," Phys. Chem. Chem. Phys. 17, 31582-31591 (2015).

30 See Supplemental Material at [URL will be inserted by publisher] for the supporting pdf document.

31 Frank Eckert, Peter Pulay, and Hans-Joachim Werner, "Ab initio geometry optimization for large molecules," Journal of Computational Chemistry 18, 1473-1483 (1997).

32 Eduardo Machado-Charry, Laurent Karim Béland, Damien Caliste, Luigi Genovese, Thierry Deutsch, Normand Mousseau, and Pascal Pochet, "Optimized energy landscape exploration using the ab initio based activationrelaxation technique," The Journal of Chemical Physics 135, 034102 (2011).

33 John E. Northrup and J. Neugebauer, "Theory of GaN $(10 \overline{1} 0)$ and (112̄0) surfaces," Phys. Rev. B 53, R10477-R10480 (1996).

34 Alessio Filippetti, Vincenzo Fiorentini, Giancarlo Cappellini, and Andrea Bosin, "Anomalous relaxations and chemical trends at iii-v semiconductor nitride nonpolar surfaces," Phys. Rev. B 59, 8026-8031 (1999).

35 M.-H Tsai, O.F Sankey, K.E Schmidt, and I.S.T Tsong, "Electronic structures of polar and nonpolar GaN surfaces," Materials Science and Engineering: B 88, $40-46$ (2002).

36 David Segev and Chris G. Van de Walle, "Surface reconstructions on InN and GaN polar and nonpolar surfaces," Surface Science 601, L15 - L18 (2007). 
37 Stefan Goedecker, Frédéric Lançon, and Thierry Deutsch, "Linear scaling relaxation of the atomic positions in nanostructures," Phys. Rev. B 64, 161102 (2001).

38 Mitsuo Takeda and Jun Suzuki, "Crystallographic heterodyne phase detection for highly sensitive lattice-distortion measurements," J. Opt. Soc. Am. A 13, 1495-1500 (1996).

39 M.J. Hÿtch, E. Snoeck, and R. Kilaas, "Quantitative measurement of displacement and strain fields from hrem micrographs," Ultramicroscopy 74, 131 - 146 (1998).

40 J.L. Rouvière and E. Sarigiannidou, "Theoretical discussions on the geometrical phase analysis," Ultramicroscopy 106, 1 - 17 (2005).
41 S.J. Leake, V. Favre-Nicolin, E. Zatterin, M-I. Richard, S. Fernandez, G. Chahine, T. Zhou, P. Boesecke, H. Djazouli, and T.U. Schülli, "Coherent nanoscale X-ray probe for crystal interrogation at ID01, ESRF - the European Synchrotron," Materials \& Design 119, 470-471 (2017).

${ }^{42}$ Lijun $\mathrm{Wu}$, Yimei Zhu, and J. Tafto, "Picometer accuracy in measuring lattice displacements across planar faults by interferometry in coherent electron diffraction," Phys. Rev. Lett. 85, 5126-5129 (2000).

43 Shoucong Ning, Takeshi Fujita, Anmin Nie, Ziqian Wang, Xiandong $\mathrm{Xu}$, Jianghua Chen, Mingwei Chen, Shuhuai Yao, and Tong-Yi Zhang, "Scanning distortion correction in STEM images," Ultramicroscopy 184, 274-283 (2018). 